\title{
Impact of body-mass factors on setup displacement during pelvic irradiation in patients with lower abdominal cancer
}

\author{
Wei-Chieh Wu' ${ }^{1}$, Yi-Ru Chang ${ }^{1}$, Yo-Liang Lai ${ }^{1}$, An-Cheng Shiau ${ }^{1,3,5}$, Ji-An Liang ${ }^{1,2}$, \\ Chun-Ru Chien ${ }^{1,2}$, Yu-Cheng Kuo ${ }^{1}$, Shang-Wen Chen ${ }^{1,2,4}$ \\ 1 Department of Radiation Oncology, China Medical University Hospital, Taichung, Taiwan \\ ${ }^{2}$ School of Medicine, College of Medicine, China Medical University, Taichung, Taiwan \\ ${ }^{3}$ Department of Biomedical Imaging and Radiological Sciences, China Medical University, Taichung, Taiwan \\ ${ }^{4}$ School of Medicine, College of Medicine, Taipei Medical University, Taipei, Taiwan \\ ${ }^{5}$ Department of Biomedical Imaging and Radiological Sciences, National Yang-Ming University, Taipei, Taiwan
}

Radiol Oncol 2019; 53(2): 256-264.

Received 4 September 2018

Accepted 3 March 2019

Correspondence to: Dr. Shang-Wen Chen, 2nd Yuh-Der Rd, North District, Taichung City, Taiwan. Phone: 886422052121 7450; E-mail: vincent1680616@yahoo.com.tw and An-Cheng Shiau, PhD, Department of Radiation Oncology, China Medical University Hospital, Taichung, Taiwan. E-mail: shiau158@ms22.hinet.net

Disclosure: No potential conflicts of interest were disclosed.

Background. The aim of the study was investigate the impact of body-mass factors (BMF) on setup displacement during pelvic radiotherapy in patients with lower abdominal cancers.

Patients and methods. The clinical data of a training cohort composed of 60 patients with gynecological, rectal, or prostate cancer were analyzed. The daily alignment data from image-guided radiotherapy (IGRT) were retrieved. Setup errors for were assessed by systematic error (SE) and random error (RE) through the superior-inferior (SI), anteriorposterior (AP), and medial-lateral (ML) directions. Several BMFs and patient-related parameters were analyzed with binary logistic regression and receiver-operating characteristic curves. A scoring system was proposed to identify those with greater setup displacement during daily treatment. The results were validated by another cohort.

Results. A large hip lateral diameter correlated with a greater SI-SE and AP-SE, whereas a large umbilical AP diameter correlated with a greater ML-SE and ML-RE. A higher SI-RE was associated with a large hip circumference. The positive predictors for setup uncertainty were chosen to dichotomize patients into groups at high risk and low risk for setup displacement. Based on the scoring system, the adequate treatment margins for the SI direction in the highand low-risk groups were $5.4 \mathrm{~mm}$ and $3.8 \mathrm{~mm}$, whereas those for the $\mathrm{ML}$ direction were $8.2 \mathrm{~mm}$ and $4.2 \mathrm{~mm}$, respectively. The validated cohort showed a similar trend.

Conclusions. Large BMFs including hip lateral diameter, hip circumference, and umbilical AP diameter are associated with greater setup uncertainty. Based on the scores, IGRT or required treatment margins can be adapted for patients with high risk features.

Key words: body-mass factors; setup displacement; image-guided radiotherapy; lower abdominal cancers

\section{Introduction}

Cancers in the lower abdomen, such as prostate, rectal, and gynecological cancers, are common malignancies worldwide. ${ }^{1}$ Pelvic irradiation is frequently used in the treatment of these patients. However, acute or chronic gastrointestinal or genitourinary toxicities might jeopardize the treatment compliance and quality of life in some patients. As a modern technique such as intensity-modulated radiation therapy (IMRT) is capable of dose painting and has been implemented to deliver tumoricidal doses to the target volume while sparing the adjacent normal tissues ${ }^{2}$, setup accuracy is more critical to minimize deviation from the planned target. Currently, treatment alignment is carried out 
by lining up skin markers with an equipped laser system. In some circumstances however, the effectiveness of skin alignment might be offset because the exact external position does not always match the internal anatomy accurately. The uncertainties, leading to inadequate dosage to the tumors or untoward toxicities, can be attributed to setup errors or organ motion.

Image-guided radiation therapy (IGRT) using kilo-voltage imaging, and cone beam computed tomography (CBCT) have been widely applied to quantify geometrical uncertainties for daily treatment setup ${ }^{3,4}$; however, they are not feasible for widespread use due to the increasing treatment time, cost, and daily dose to the patients ${ }^{5}$, the technique and frequency of using IGRT should be adjusted based on the clinical conditions. In some developing countries, not all cancer patients requiring radiotherapy are able to receive adequate treatment. ${ }^{6}$ Particularly, patients who can undergo weekly or daily IGRT were limited even in some institutes where patient load was huge. ${ }^{7}$ In Europe, IGRT was available in only $49 \%$ of all linear accelerators. ${ }^{8}$ Therefore, tailored use of IGRT for patients with a high risk of setup displacement is an important issue, particularly in countries or institutions where IGRT resources are limited.

Many studies have reported that greater margins are required for obese patients due to higher setup uncertainties., ${ }^{3,4,9-11}$ However, most studies investigated only the relationship between body mass index (BMI) and the magnitude of setup errors. The impact of patient-related parameters or body-mass factors (BMF) on setup displacement in patients receiving pelvic irradiation remains to be clarified. We hypothesized that the uncertainties can be scored according to the BMFs. Therefore, this study investigated the effect of BMFs on the magnitude of setup displacement during pelvic radiotherapy. As a result, patients with high-risk features or those who requiring large margins between the planning target volume (PTV) and clinical target volume (CTV) can be determined.

\section{Patients and methods}

\section{Patient}

This study was approved by the local Institutional Review Board (CMUH106-REC3-119).

Patients were divided into two cohorts (60 for training, 30 for validation). In the training cohort, patients with gynecological (cervix or endometrium), rectal, or prostate cancer treated with pelvic irradiation by daily IGRT between January 2012 and January 2015 at China Medical University Hospital were included. The sample size for gynecological, rectal, and prostate cancers was 20 each. The patient-related parameters and BMFs were retrieved. Staging was based on the staging system ( $7^{\text {th }}$ edition, 2010)..$^{12}$ Performance status was assessed according to the Eastern Cooperative Oncology Group criteria. The characteristics for the training cohort are listed in Table 1. Another 30 patients composed of 10 cases of each cancer type were labeled as the validation cohort.

\section{Treatment planning}

To minimize setup uncertainties as reported previously ${ }^{13,14}$, patients were immobilized by a vacuum

TABLE 1. The patient-related parameters and body-mass factors of the training cohort

\begin{tabular}{|c|c|c|c|c|}
\hline \multicolumn{2}{|c|}{ Parameters } & Number & Median & Range \\
\hline \multicolumn{2}{|l|}{ Age $(y / O)$} & & 64.5 & $38-90$ \\
\hline \multicolumn{2}{|l|}{$\mathrm{BW}(\mathrm{kg})$} & & 61 & $45.4-99.3$ \\
\hline \multicolumn{2}{|l|}{$\mathrm{BH}(\mathrm{cm})$} & & 160.6 & $142.2-177.3$ \\
\hline \multicolumn{2}{|c|}{$\mathrm{BMI}\left(\mathrm{kg} / \mathrm{m}^{2}\right)$} & & 23.7 & 17.99-35.69 \\
\hline \multicolumn{2}{|c|}{ Umbilical circumference (UC, cm) } & & 87.8 & $63.4-120.3$ \\
\hline \multicolumn{2}{|c|}{ Umbilical AP diameter (UAPD, cm) } & & 19.25 & $13.4-28.6$ \\
\hline \multicolumn{2}{|c|}{ Umbilical lateral diameter (ULD, cm) } & & 32.6 & $25-46.4$ \\
\hline \multicolumn{2}{|c|}{ Hip circumference (HC, cm) } & & 94.7 & $75-117.8$ \\
\hline \multicolumn{2}{|c|}{ Hip AP diameter (HAPD, cm) } & & 20.65 & $17.1-26.8$ \\
\hline \multicolumn{2}{|c|}{ Hip Lateral diameter (HLD, cm) } & & 35.45 & $30.6-46.4$ \\
\hline \multicolumn{2}{|c|}{ CTV circumference (CTVC, cm) } & & 93.45 & $72.8-118.3$ \\
\hline \multicolumn{2}{|c|}{ CTV AP diameter (CTVAPD, cm) } & & 20.45 & $15.1-27.9$ \\
\hline \multicolumn{2}{|c|}{ CTV lateral diameter (CTVLD, cm) } & & 35.45 & $27.2-46.5$ \\
\hline \multirow{3}{*}{ Cancer } & Rectum & 20 & & \\
\hline & Prostate & 20 & & \\
\hline & Gynecology & 20 & & \\
\hline \multirow{2}{*}{ Sex } & Female & 31 & & \\
\hline & Male & 29 & & \\
\hline \multirow{2}{*}{ ECOG PS } & 0 & 29 & & \\
\hline & $1-2$ & 31 & & \\
\hline \multirow{2}{*}{ Surgery } & - & 46 & & \\
\hline & + & 14 & & \\
\hline \multirow{2}{*}{ CCRT } & - & 25 & & \\
\hline & + & 35 & & \\
\hline
\end{tabular}

$\mathrm{BH}=$ body height; $\mathrm{BMI}=$ body mass index; $\mathrm{BW}=$ body weight; $\mathrm{CCRT}=$ concurrent chemoradiotherapy; CTV = clinical target volume; ECOG PS = Eastern Cooperative Oncology Group performance status; 
bag (VacBag, Blessing Cathay Corporation) or alpha cradle (Blessing Cathay) from the chest to the lower pelvis to enhance the accuracy of the daily treatment position. All patients were suggested to defecate before simulation and daily treatment to reduce the organ motion of the rectum. ${ }^{13}$ In addition, patients with prostate cancer were requested to drink a fixed amount of water after emptying the bladder. Computed tomographic (CT) simulation was done with patients in the supine position using a CT scanner (HiSpeed NX/i, GE Healthcare, Florida, USA). The CT images were scanned from the T12 vertebral body to $2 \mathrm{~cm}$ below the ischial tuberosities using a slice thickness of $3 \mathrm{~mm}$. External markers were made on the skin using setup lasers to facilitate an accurate daily position.

The CTV was contoured according to the radiotherapy guidelines for each cancer. Generally, the CTV was expanded by 0.7 to $1.5 \mathrm{~cm}$ to create the PTV for organ motion and setup errors. All patients underwent IMRT planning using 6 or 10 MV photons. All plans were calculated using a commercial radiation treatment planning system (Eclipse, Varian Medical Systems Inc, Palo Alto, California, USA).

\section{Anthropometric measurements of body- mass factors}

The studied BMFs included body weight (BW), body height $(\mathrm{BH}), \mathrm{BMI}$, umbilical circumference (UC), umbilical anterior-posterior diameter (UAPD), umbilical lateral diameter (ULD), hip circumference (HC), hip anterior-posterior diameter (HAPD), and hip lateral diameter (HLD). In addition, CTV circumference, CTV anterior-posterior diameter, and CTV lateral diameter were defined at the center of the CTV.

$\mathrm{BW}$ and $\mathrm{BH}$ were recorded from pretreatment evaluations. The BMI was calculated as the weight in kilograms divided by height in meters squared according to the definition of the World Health Organization. ${ }^{15}$ Circumferences and diameters were measured according to the $\mathrm{CT}$ images from the simulation. The UC, UAPD, and ULD were calculated at the level of the umbilicus. The $\mathrm{HC}$, HAPD, and HLD were obtained at the top of the femoral head. Generally, BMFs of the hip measured at the top of the femoral head match the widest level of the hip. Representative images for definition of the BMFs are illustrated in Figure 1.
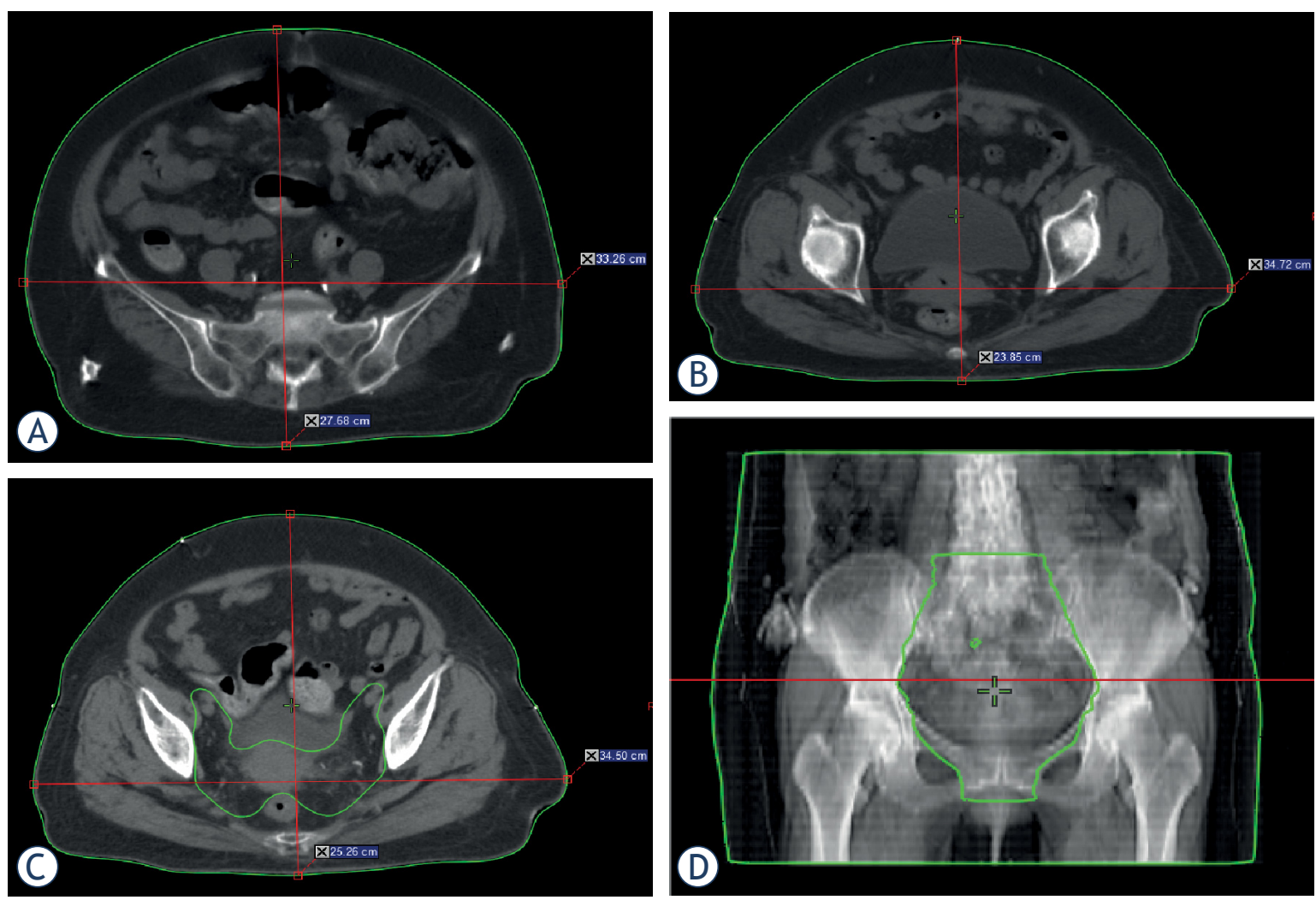

FIGURE 1. An example of body-mass factor measurement in a patient with rectal cancer. (A) umbilical circumference, umbilical anterior-posterior (AP) diameter, umbilical lateral diameter; (B) hip circumference, hip AP diameter, hip Lateral diameter; (C) clinical target volume (CTV) circumference, CTV AP diameter, CTV lateral diameter; (D) level of CTV center. 


\section{Daily treatment verification and setup displacement}

All patients underwent pelvic radiotherapy with a daily dose of $1.8 \mathrm{~Gy}$. The minimum prescribed dose was $45 \mathrm{~Gy}$ in 25 fractions. IGRT was carried out with a Varian Clinac iX linear accelerator (Varian Medical Systems) equipped with online on- board imaging (OBI) and CBCT function. Before daily treatment, patients were positioned on the couch according to the alignment markers drawn on the body during the simulation. On-line two-dimensional kilovoltage $(\mathrm{kV})$ images were taken daily or three-dimensional kV CBCT images were obtained weekly to verify the setup accuracy. The images were registered to the digitally reconstructed radiographs from the treatment planning CT images and compared to the planning CT by aligning with the bony landmarks. As a result, the irradiated field could be adjusted by shifting the couch. The quantification of image correction was recorded in the superior-inferior (SI), anterior-posterior (AP), and medial-lateral (ML) directions, and couch rotation (CR). The on-line calibrated images were confirmed by physicians if the displacement of any translational direction was more than $3 \mathrm{~mm}$.

As described previously ${ }^{13,16}$, setup errors for each patient were assessed by systematic errors (SE) and random errors (RE) through the 4 directions. The mean and standard deviation (SD) of each translational displacement were documented for the individual. The population SE was calculated as the SD of the mean setup correction for each patient. The population RE was determined by calculating the root mean square of the SD of the setup displacement. ${ }^{17,18}$ The margins from the CTV to PTV were calculated via a formula described by Van Herk et al. ${ }^{19,20}$, in which the suggested margin was 2.5 SE + 0.7 RE to ensure that the minimum dose to the CTV is $95 \%$ for $90 \%$ of patients.

\section{Statistical analysis}

The training cohort was stratified into low- and high- setup displacement groups according to the median values of the errors through the three translational directions. Pearson's correlation was performed to model the possibility of linear association between individual setup errors and BMFs. Because the dependent variable was dichotomous in this study, binary logistic regression was used to examine the effects of continuous or categorical variables across the patient-related parameters or BMFs associated with higher SEs or REs. Using the optimal cutoffs of the parameters through receiver-operating characteristic curve analysis a scoring system was proposed according to the predictors identified from the results of binary ogistic regression analysis. Accordingly, the patients were dichotomized to high- and low-risk groups and the required CTV-PTV margins were calculated for each group. To differentiate the risk groups, optimal cutoffs of the BMFs in predicting the setup errors were chosen through receiver-operating characteristic (ROC) curve analysis. To confirm the validity, the scoring system was applied to test the validation cohort. The magnitude of the setup displacement between groups was examined by the chi-square test. In this study, $P<.05$ was considered statistically significant. All statistical analyses were performed using IBM SPSS version 22.0 (IBM, Armonk, New York, USA).

\section{Results}

In the training cohort, a total of 1976 setup images including the CBCT or OBI were analyzed. As listed in Table 2, the population SE / REs were 1.1 / $2.6 \mathrm{~mm}, 1.1 / 2.0 \mathrm{~mm}$, and $1.9 / 5.0 \mathrm{~mm}$ in the SI, $\mathrm{AP}$ and ML directions, respectively. The SEs and RE of CR were 0.23 and 0.44 degrees. According to Van Herk's formula ${ }^{19,20}$, the suggested CTV- PTV margins for minimizing setup uncertainties were 4.5, 4.0 and $8.1 \mathrm{~mm}$ in the AP, ML and SI directions, respectively.

As shown in Figure 2, a linear relationship existed between the individual setup errors and certain BMFs, especially between ML-SE and umbilical AP diameter and between ML-RE and umbilical AP diameter (Coefficient: 0.536 and 0.604, respectively). Table 3 shows the results of univariate and multivariate analyses of the binary logistic regression in the training cohort. Female gender was associated with increasing uncertainties of ML-SE

TABLE 2. The population SE/RE and calculated PTV margins of training cohort

\begin{tabular}{lccc}
\hline Direction & $\begin{array}{c}\text { Population } \\
\text { SE }\end{array}$ & $\begin{array}{c}\text { Population } \\
\text { RE }\end{array}$ & $\begin{array}{c}\text { PTV } \\
\text { margin } \\
\text { (cm) }\end{array}$ \\
\hline Superior-Inferior $(\mathrm{cm})$ & 0.11 & 0.26 & 0.45 \\
Anterior-Posterior $(\mathrm{cm})$ & 0.11 & 0.20 & 0.40 \\
Medial-Lateral $(\mathrm{cm})$ & 0.19 & 0.50 & 0.81 \\
Couch rotation (degree) & 0.23 & 0.44 & \\
\hline
\end{tabular}

$\mathrm{RE}=$ random error; $\mathrm{PPTV}=$ phantom planning target volume; $\mathrm{SE}=$ systematic error 

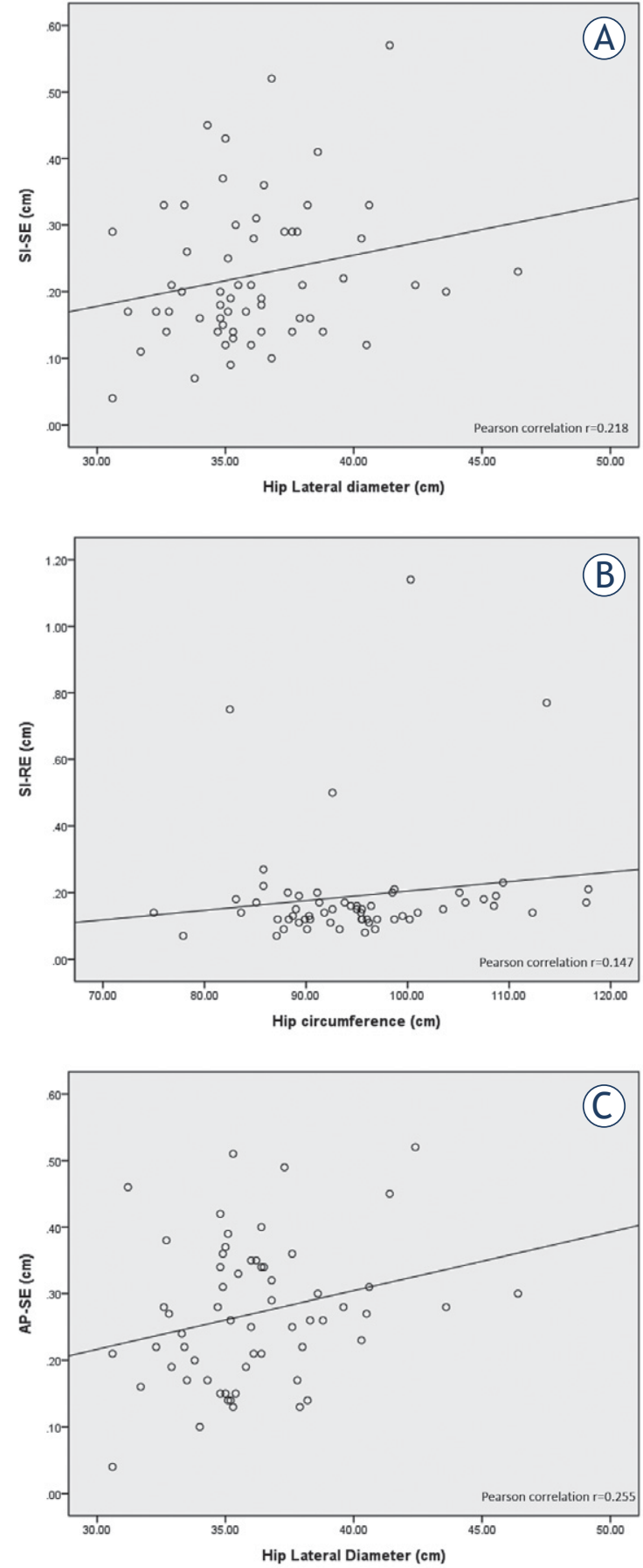

only in univariate analysis. We found that a large HLD correlated with a greater SI-SE and AP-SE $(P$ $=0.036$ and 0.044$)$, whereas a large UAPD correlated with a greater ML-SE and ML-RE $(P=0.021$ and 0.001). In addition, a higher SI-RE was associated with a large $\mathrm{HC}(P=0.008)$. Furthermore, patients without previous surgery were vulnerable to a greater CR-RE $(P=0.003)$.
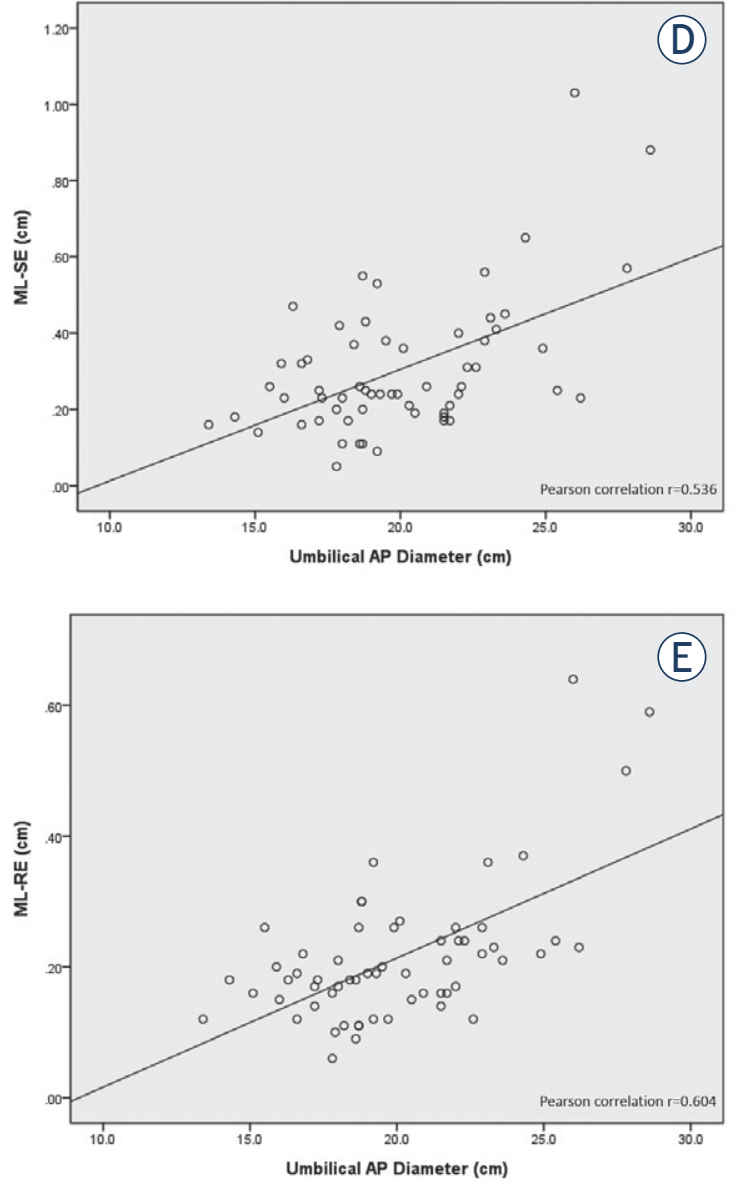

FIGURE 2. A linear relationship between individual setup errors and body-mass factors. (A) SI-SE and hip lateral diameter; (B) SIRE and hip circumference; (C) AP-SE and hip lateral diameter; (D) ML-SE and umbilical AP diameter; (E) ML-RE and umbilical AP diameter.

$\mathrm{AP}-\mathrm{SE}=$ systemic error of anterior-posterior direction; $\mathrm{ML}-\mathrm{RE}=$ random error of medial-lateral direction; ML-SE = systemic error of medial-lateral direction; SI-RE = random error of superior-inferior direction; SI-SE = systemic error of superior-inferior direction

To differentiate the risk groups, the ROC curves showed the optimal cutoffs of the BMFs in predicting the setup errors as illustrated in Figure 3. The values were $36.5 \mathrm{~cm}$ for HLD and $102.3 \mathrm{~cm}$ for HC in the SI direction, $34.5 \mathrm{~cm}$ for HLD in the AP direction, and $22.1 \mathrm{~cm}$ for UAPD in the ML direction. A scoring system to stratify the risk groups was proposed according to the scores of these predictors. In the SI direction, the two BMFs (HLD and $\mathrm{HC}$ ) were utilized to score the risk of setup errors. Each positive predictor scored one point and accordingly patients were dichotomized into groups at high risk and low risk (0 versus 1-2 points) for setup errors. In the AP and ML direction, patients were grouped according to the HLD and UAPD, respectively. Based on the scores, the required 
TABLE 3. Univariate and multivariate of patient related parameters and BMFs for setup displacement

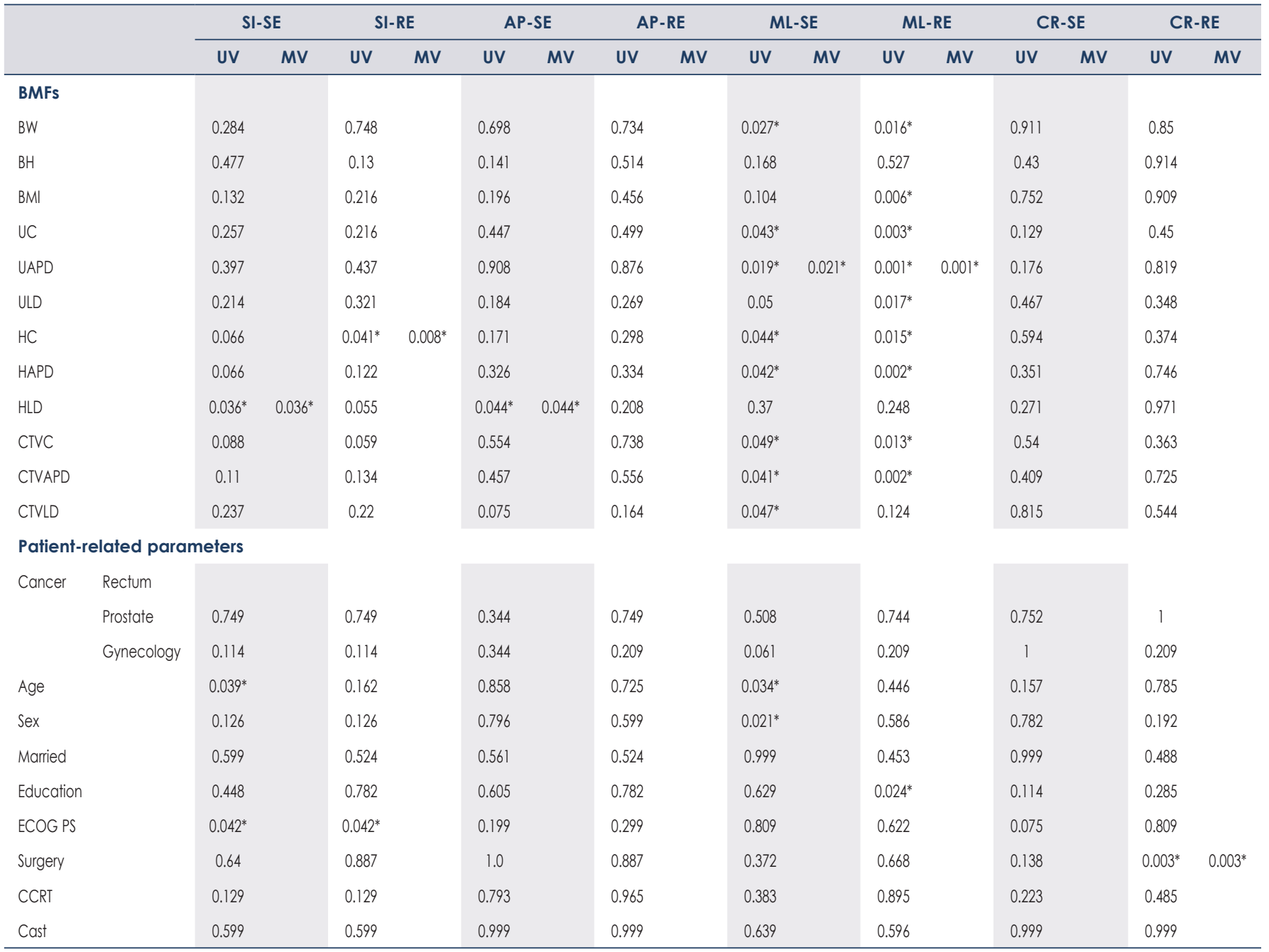

$\mathrm{AP}=$ anterior-posterior: $\mathrm{BH}=$ body height: $\mathrm{BMFs}=$ body mass factors: $\mathrm{BMI}=$ body mass index: $\mathrm{BW}=$ body weight: $\mathrm{CCRT}=$ concurrent $\mathrm{chemoradiotherapy}$ : $\mathrm{CR}=\mathrm{couch}$ rotation: CTVAPD = CTV anterior-posterior diameter; CTVC = CTV circumference; CTVLD = CTV lateral diameter; ECOG PS = Eastern Cooperative Oncology Group performance status; $\mathrm{HAPD}=$ hip anterior-posterior diameter; $\mathrm{HC}=$ hip circumference; $\mathrm{HLD}=$ hip lateral diameter; $\mathrm{ML}=$ medial-lateral; $\mathrm{MV}=$ multivariate; $\mathrm{RE}=$ random error; $\mathrm{SE}=\mathrm{systematic}$ error; $\mathrm{SI}$ = superior-inferior; RE = random error; UAPD = umbilical anterior-posterior diameter; UC = umbilical circumference; ULD = umbilical lateral diameter; UV = univariate

PTV-CTV margin for the SI direction in the highand low-risk groups were $5.4 \mathrm{~mm}$ and $3.8 \mathrm{~mm}$, whereas those for the ML direction were $8.2 \mathrm{~mm}$ and $4.2 \mathrm{~mm}$, respectively (Table 4 ).

In the validation cohort, a total of 959 setup images were retrieved. There was no difference between the training and validation cohorts regarding gender or BMI (gender 1:1, median BMI 25.3). The population SE / REs were 1.0 /1.6 mm, 1.2 /2.4 $\mathrm{mm}$, and $1.6 / 2.8 \mathrm{~mm}$ in the SI, AP, and ML directions, respectively. As listed in Table 5, a similar trend of a greater population RE and required PTV-CTV margins could be found when using the same scoring criteria to classify the low- and highrisk groups.

\section{Discussion}

This is the first study to report the impact of imagederived BMFs and other patient-related parameters to score the magnitude of setup displacement during pelvic radiotherapy in patients with lower abdominal cancers. Our results disclosed that certain BMFs have a significant effect on setup errors in specific translational directions. The displacement in the SI direction was greater in patients with higher HC and HLD. A higher HLD and UAPD were associated with greater shifts in the AP and ML directions, respectively. Furthermore, a scoring system for the high-risk group was proposed and validated. 

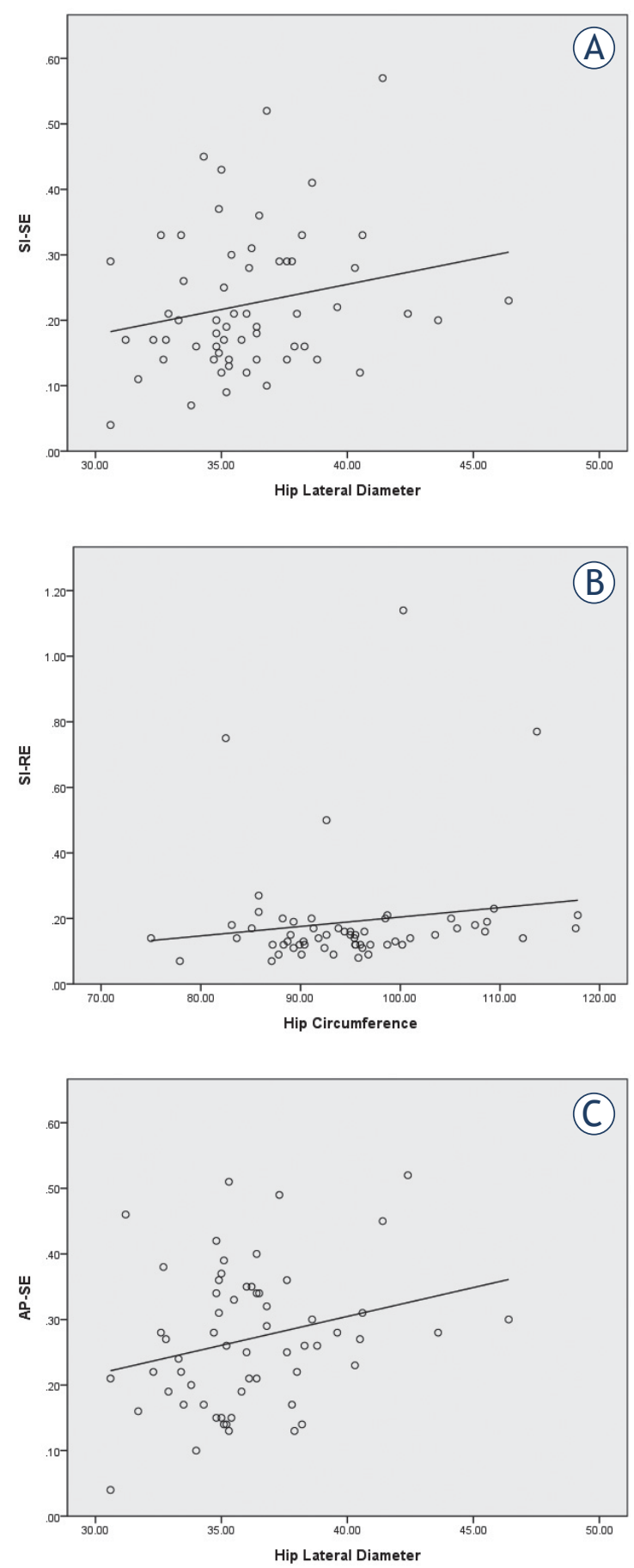

Wong et al. investigated the correlation between BMI and daily setup deviation in 117 patients who received IGRT for prostate cancer. ${ }^{9}$ They reported that setup shifts greater than $10 \mathrm{~mm}$ in the ML direction increase significantly as the BMI increases, with a $1.3 \%$ shift for those with normal body weight to a $21.2 \%$ shift for those with severe obesity. Strong correlations were found between
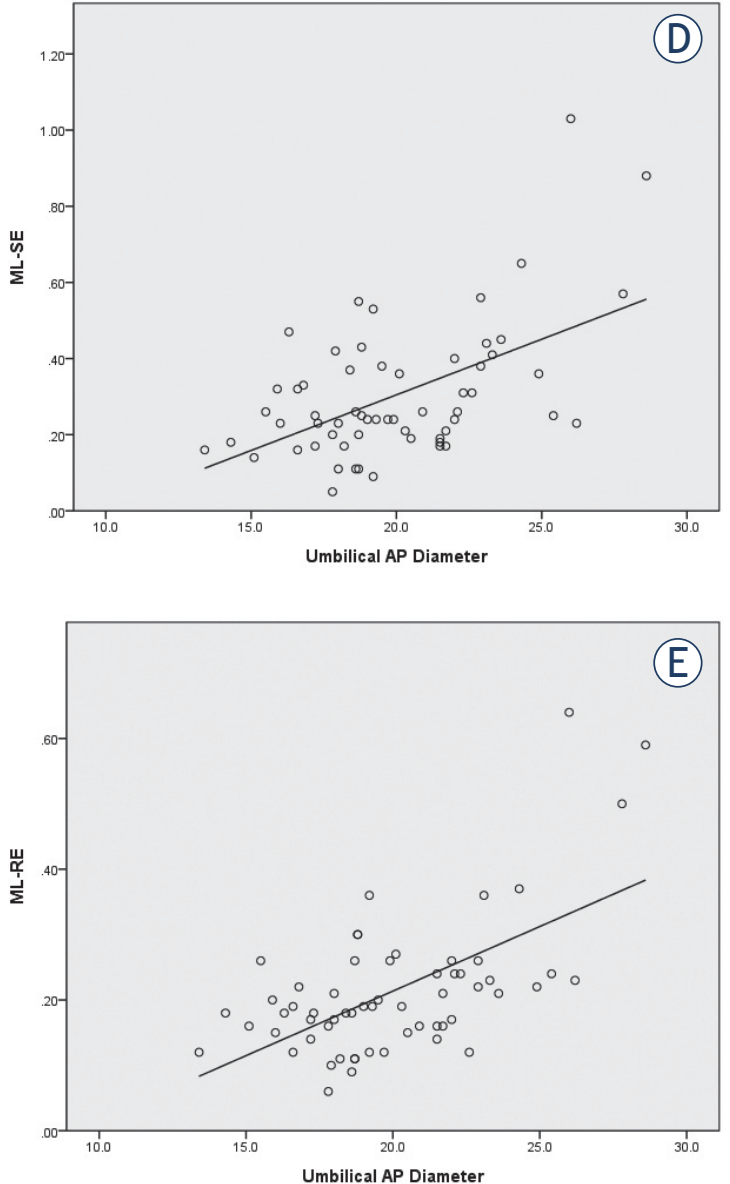

FIGURE 3. Receiver operating characteristic curves for assessing the optimal cutoffs of body-mass factors. (A) systemic errors of superior-inferior direction, AUC 0.638; (B) random errors of superior-inferior direction, AUC 0.644; (C) systemic errors of anterior-posterior direction, AUC 0.617; (D) systemic errors of medial-lateral direction, AUC 0.701; (E) systemic errors of medial-lateral direction, AUC 0.746 .

some BMFs such as subcutaneous adipose-tissue thickness, BMI, body weight, and the SDs of daily displacements in the ML direction. Lin et al. demonstrated that mean absolute shifts in three translational directions positively correlated with the BMI in 30 patients with endometrial cancer treated with adjuvant pelvic IMRT ${ }^{10}$. Kim et al. revealed that the mean shifts in the ML direction were $0.9 \mathrm{~mm}$ for those with a BMI $\geq 30$ and $0.1 \mathrm{~mm}$ for those with a BMI $<30(P=0.02)^{3}$. In addition, Bray et al. revealed that obese patients had larger mean displacements and REs in the ML direction. ${ }^{4}$ Undoubtedly, some BMFs have a great impact on setup uncertainties. However, a scoring system is required to identify high-risk patients for daily IGRT or to employ a large PVT-CTV margin. 
In this study, the required CTV-PTV margins for all populations in the SI, AP, and ML directions were $4.5,4.0$ and $8.1 \mathrm{~mm}$, respectively. The greatest setup uncertainties were present in the ML direction, similar to previous studies. ${ }^{3,4}$ Although daily IGRT could reduce setup variations in patients receiving pelvic irradiation ${ }^{3,4}$, it is not always accessible due to limited facilities in some institutions as well as concerns about the increased daily dose to patients. ${ }^{8}$ Based on our scores, we could adapt the required PTV-CTV margins $(5.4 \mathrm{~mm}$ for SI and $8.2 \mathrm{~mm}$ for ML) for patients with high risk features. Certainly, the clinical validity of the scoring system needs to be verified by external validation.

Laaksomaa et al. ${ }^{21}$ investigated the influence of gender on setup uncertainties in patients with pelvic cancers and found larger SEs and REs in women. As a result, women required greater PTV-CTV margins in the three translational directions. They also suggested that the difference in the amount of subcutaneous fat between sexes might contribute to this difference. In multivariate analysis in our study however, female gender did not impact the setup uncertainty. The discrepancy could be attributed to the fact that various distributions of accumulated adipose had been included in the BMF analyses, and consequently the impact of gender was diluted.

In several studies, the setup uncertainties were larger in obese patients despite the use of immobilization devices. . $^{3,4-11}$ Particularly, obesity has a negative influence on toxicity for prostate cancer patients treated with 3-dimensional radiotherapy without IGRT.22 Therefore, for prostate cancer patients who cannot be managed with IGRT or surgical treatment, a sophisticated guidance for PTV-CTV margin to reduce setup uncertainty during radiotherapy is required. Currently, obesity is usually determined by BMI alone. However, there are two kinds of obesity, the central and peripheral types, depending on the area of fat accumulation. The BMI is not able to distinguish entirely central obesity from the peripheral type. ${ }^{23}$ Based on the external surface markers on the belly, the type of obesity might influence the setup errors because the skin folds would be more movable in central obesity. To overcome this limitation, this study retrieved the UC, HC, and diameters of in the AP and lateral directions from the simulation $\mathrm{CT}$, which could include the effects of different types of obesity. Thus, our data evidenced that the abdominal or hip circumferences and diameters are more effective in predicting greater setup uncertainties compared with the BMI.
TABLE 4. Population SE/RE and adequate PTV margins according to scoring system by significant associated factors in three translational directions in training cohort

\begin{tabular}{|c|c|c|c|c|c|c|}
\hline \multicolumn{2}{|c|}{ Direction } & \multicolumn{2}{|c|}{$\begin{array}{l}\text { Population SE } \\
\qquad(\mathrm{cm})\end{array}$} & \multicolumn{2}{|c|}{$\begin{array}{l}\text { Population RE } \\
(\mathrm{cm})\end{array}$} & \multirow{2}{*}{$\begin{array}{c}\text { PTV margin } \\
(\mathrm{cm})\end{array}$} \\
\hline \multirow{2}{*}{ SI } & High risk (1-2) & 0.12 & \multirow{2}{*}{$p=0.016^{*}$} & 0.33 & \multirow{2}{*}{$p=0.016^{*}$} & \\
\hline & Low risk (0) & 0.09 & & 0.20 & & 0.38 \\
\hline \multirow{2}{*}{ AP } & High risk & 0.10 & \multirow{2}{*}{$p=0.044^{*}$} & 0.20 & \multirow{2}{*}{$p=0.236$} & 0.40 \\
\hline & Low risk & 0.10 & & 0.18 & & 0.38 \\
\hline \multirow{2}{*}{$M L$} & High risk & 0.23 & \multirow{2}{*}{$p=0.004^{*}$} & 0.34 & \multirow{2}{*}{$p=0.005^{*}$} & 0.82 \\
\hline & Low risk & 0.11 & & 0.19 & & 0.42 \\
\hline
\end{tabular}

* = statistical significance

$\mathrm{AP}=$ anterior-posterior; $\mathrm{ML}=$ medial-lateral; $\mathrm{PTV}=$ planning target volume; $\mathrm{RE}=$ random error; $\mathrm{SE}=$ systematic error; $\mathrm{SI}$ = superior-inferior

TABLE 5. The Population SE/RE and adequate PTV margins according to scoring system in validation cohort

\begin{tabular}{|c|c|c|c|c|c|c|}
\hline \multicolumn{2}{|c|}{ Direction } & \multicolumn{2}{|c|}{$\begin{array}{l}\text { Population SE } \\
(\mathrm{cm})\end{array}$} & \multicolumn{2}{|c|}{$\begin{array}{l}\text { Population RE } \\
(\mathrm{cm})\end{array}$} & \multirow{2}{*}{$\begin{array}{c}\text { PTV margin } \\
\text { (cm) } \\
0.44\end{array}$} \\
\hline \multirow{2}{*}{ SI } & High risk (1-2) & 0.13 & \multirow{2}{*}{$p=0.358$} & 0.17 & \multirow{2}{*}{$p=0.225$} & \\
\hline & Low risk (0) & 0.07 & & 0.14 & & 0.27 \\
\hline \multirow{2}{*}{ AP } & High risk & 0.12 & \multirow{2}{*}{$p=0.213$} & 0.26 & \multirow{2}{*}{$p=0.054$} & 0.48 \\
\hline & Low risk & 0.12 & & 0.18 & & 0.42 \\
\hline \multirow{2}{*}{$M L$} & High risk & 0.23 & \multirow{2}{*}{$p=0.195$} & 0.45 & \multirow{2}{*}{$p=0.004^{*}$} & 0.90 \\
\hline & Low risk & 0.11 & & 0.20 & & 0.41 \\
\hline
\end{tabular}

* = statistical significance

$\mathrm{AP}=$ anterior-posterior; $\mathrm{ML}=$ medial-lateral; $\mathrm{PTV}=$ planning target volume; $\mathrm{RE}=$ random error; $\mathrm{SE}=$ systematic error; $\mathrm{SI}=$ superior-inferior

This study was subject to several limitations. First, the circumferences and diameters of the patients were collected retrospectively from CT images instead of direct measurement of the girdle of the bodies. Although the mean deviation between the two methods was less than $5 \%$ according to a previous comparison test, the concordance of the two approaches should be assessed further. Second, the strength of the validation test was limited because of the small sample size. However, a trend of a greater RE in the high-risk group could be found among the three translational directions. Finally, organ motion or tumor regression may affect daily treatment accuracy, and the values across various cancers might be different. Our study did not explore the impact of these two factors through daily $\mathrm{CBCT}$, as well as weekly dosimetric changes. Future studies should enroll patients prospectively and evaluate subsequent dosimetric changes according to evolution of the BMFs. Furthermore, external validation is needed to facilitate widespread utility of the scoring system. 


\section{Conclusions}

Several BMFs including the HLD, HC, and UAPD are associated with greater setup uncertainties in patients receiving pelvic irradiation for lower abdominal cancers. Based on the scores, IGRT can be suggested for patients with high risk features, or required PTV margins could be adapted for patients who cannot be managed with IGRT.

\section{Authors' contributions}

WC Wu and SW Chen were responsible for design of the study, acquisition of data, analysis and interpretation of data, and drafting the article. YR Chang and YL Lai help to collect the clinical data. JA Liang, CR Chien, YC Kuo, and AC Shiau provided some intellectual content. SW Chen approved the version to be submitted.

\section{Acknowledgements}

We thank for the approval of Institutional Review Board of China Medical University Hospital.

\section{References}

1. Jemal A, Siegel R, Ward E, Hao Y, Xu J, Thun MJ. Cancer statistics, 2009. CA Cancer J Clin 2009; 59: 225-49. doi: 10.3322/caac.20006

2. Hasselle MD, Rose BS, Kochanski JD, Nath SK, Bafana R, Yashar CM, et al. Clinical outcome of intensity-modulated pelvic radiation therapy for carcinoma of the cervix. Int J Radiat Oncol Biol Phys 2011; 80: 1436-45. doi: 10.1016/j.ijrobp.2010.04.041

3. Kim H, Beriwal S, Huq MS, Kannan N, Shukla G, Houser C: Evaluation of set-up uncertainties with daily kilovoltage image guidance in external beam radiation therapy for gynaecological cancers. Clin Oncol (R Coll Radiol) 2012 24: 39-45. doi: 10.1016/j.clon.2011.09.007

4. Bray TS, Kaczynski A, Albuquerque K, Cozzi F, Roeske JC. Role of image guided radiation therapy in obese patients with gynecologic malignancies. Pract Radiat Oncol 2013; 3: 249-55. doi: 10.1016/j.prro.2012.09.001

5. Bujold A, Craig T, Jaffray D, Dawson LA. Image-guided radiotherapy: Has it influence patient outcomes? Sem Radiat Oncol 2012; 50: 50-61. doi: 10.1016/j.semradonc.2011.09.001

6. Zubizarreta EH, Fidarova E, Healy B, Rosenblatt E. Need for radiotherapy in low and middle income countries - the silent crisis continues. Clin Oncol ( $R$ Coll Radiol) 2015; 27: 107-14. doi: 10.1016/j.clon.2014.10.006

7. Deshpande S, Dhote DS, Kumar R, Naidu S, Sutar A, Kannan V. Use of image guided radiation therapy techniques and imaging dose measurement at Indian hospitals: A survey. J Med Phys 2015; 40: 220-5. doi: 10.4103/09716203.170788

8. Grau C, Defourny N, Malicki J, Dunscombe P, Borras JM, Coffey M, et al. The impact of cancer incidence and stage on optimal utilization of radiotherapy: Methodology of a population based analysis by the ESTRO-HERO project. Radiother Oncol 2014; 112: 155-64. doi: 10.1016/j.radonc.2014.08.029
9. Wong JR, Gao Z, Merrick S, Wilson P, Uematsu M, Woo K, et al. Potentia for higher treatment failure in obese patients: correlation of elevated body mass index and increased daily prostate deviations from the radiation beam isocenters in an analysis of 1,465 computed tomographic images. Int J Radiat Oncol Biol Phys 2009; 75: 49-55. doi: 10.1016/j.jirobp.2008.07.049

10. Lin LL, Hertan L, Rengan R, Teo BK: Effect of body mass index on magnitude of setup errors in patients treated with adjuvant radiotherapy for endometrial cancer with daily image guidance. Int J Radiat Oncol Biol Phys 2012; 83: 670-5. doi: 10.1016/j.ijrobp.2011.07.026

11. Lai YL, Yu CY, Liang JA, Chen SW. Impact of the body-mass factors on set-up displacement in patients treated with pelvic irradiation for gynecological cancer with daily on-line Image guidance. Therapeut Radiol Oncol 2014; 21: 21-30.

12. American Joint Committee on Cancer. AJCC Cancer Staging Manual. 7th edition. New York. Springer Verlag; 2010

13. Melancon AD, Kudchadker RJ, Amos R, Johnson JL, Zhang Y, Yu ZH. Patientspecific and generic immobilization devices for prostate radiotherapy. Int/ J Med Phs Clin Engin Radiat Oncol 2013; 2: 125-32. doi: 10.4236/ijmpcero.2013.24017

14. Benedict SH, Yenice KM, Followill D, Galvin JM, Hinson W, Kavanagh B. Stereotactic body radiation therapy: the report of AAPM Task Group 101 Med Phys 2010; 37: 4078-101. doi: 10.1118/1.3438081

15. Waxman A; World Health Assembly. WHO global strategy on diet, physical activity and health. Food Nutr Bull 2004; 25: 292-302. doi $10.1177 / 156482650402500310$

16. Lai YL, Yang SN,Liang JA, Wang YC, Yu CY, Su CH, et al. Impact of body-mass factors on setup displacement in patients with head and neck cancer treated with radiotherapy using daily on-line image guidance. Radiat Oncol 2014; 9: 19. doi: 10.1186/1748-717X-9-19

17. Stroom JC, Heijman BJ. Geometrical uncertainties, radiotherapy planning margins, and the ICRU-62 report. Radiother Oncol 2002; 64: 75-83. doi: $10.1016 / \mathrm{S} 0167-8140(02) 00140-8$

18. Remeijer P, Geerlof E, Ploeger L, Gilhuijs K, van Herk M, Lebesque JV. 3-D portal image analysis in clinical practice: An evaluation of 2-D and 3-D analysis techniques as applied to 30 prostate cancer patients. Int J Radiat Oncol Biol Phys 2000; 46: 1281-90. doi: 10.1016/S0360-3016(99)00468-X

19. van Herk M, Remeijer P, Rasch C, Lebesque JV. The probability of correct target dosage: Dose-population histograms for deriving treatment margins in radiotherapy. Int J Radiat Oncol Biol Phys 2000; 47: 1121-35. doi: 10.1016/j. ijrobp.2011.09.010

20. Van Herk M. Errors and margins in radiotherapy. Semin Radiat Oncol 2004; 14: 52-64. doi: $10.1053 / \mathrm{j}$.semradonc.2003.10.003

21. Laaksomaa M, Kapanen M, Tulijoki T, Peltola S, Hyödynmaa S, KellokumpuLehtinen PL. Evaluation of overall setup accuracy and adequate setup margins in pelvic image-guided radiotherapy: comparison of the male and female patients. Med Dosim 2014; 39: 74-8. doi: 10.1016/j.meddos.2013.09.009

22. Dieperink KB ${ }^{1}$, Hansen S, Wagner L, Johansen $C$, Andersen KK, Hansen $\mathrm{O}$. Living alone, obesity and smoking: important factors for quality of life after radiotherapy and androgen deprivation therapy for prostate cancer. Acta Oncol. 2012; 51: 722-9. doi: 10.3109/0284186X.2012.682627.

23. Ko GT, Tang JS, Chan JC. Worsening trend of central obesity despite stable or declining body mass index in Hong Kong Chinese between 1996 and 2005. Eur J Clin Nutr 2010; 64: 549-52 doi:10.1038/ejcn.2010.49 